\title{
Alterations of proliferative and differentiation potentials of human embryonic stem cells during long-term culture
}

\author{
Yong Bin Park ${ }^{1,2}$, Yoon Young Kim², \\ Sun Kyung $\mathrm{Oh}^{2,3}$, Sun Gan Chung, \\ Seung-Yup $\mathrm{Ku}^{2,3}$, Seok Hyun $\mathrm{Kim}^{2,3}$, \\ Young Min Choi ${ }^{2,3,4}$ and Shin Yong Moon ${ }^{2,3}$ \\ ${ }^{1}$ Central Research Institute \\ Sam Jin Pharm. Co. Ltd. \\ Hwasung 445-746, Korea \\ ${ }^{2}$ Institute of Reproductive Medicine and Population \\ Medical Research Center \\ ${ }^{3}$ Department of Obstetrics and Gynecology \\ Seoul National University College of Medicine \\ Seoul 110-799, Korea \\ ${ }^{4}$ Corresponding author: Tel, 82-2-2072-2385; \\ Fax, 82-2-744-8945; E-mail, ymchoi@ snu.ac.kr
}

Accepted 26 December 2007

Abbreviations: bFGF, basic fibroblast growth factor; EB, embryoid body; hESCs, human embryonic stem cells; HNF, hepatocyte nuclear factor; ICM, inner cell mass; SSEA, stage specific embryonic antigen

\begin{abstract}
Human embryonic stem cells (hESCs) are considered to be able to stably maintain their characteristics in vitro for prolonged periods, but we had previously encountered changes in proliferative ability and differentiation potential during extended culture of hESCs. Therefore, we investigated the proliferative ability and differentiation potential of hESCs during long-term culture. The hESCs, SNUhES3, were used to analyze population-doubling time, proliferation rate and differentiation potential. We classified hESCs into three groups according to culture period. Ten colonies of hESCs for each group were daily measured colony area and population-doubling time was assessed by the changes of colony area. Proliferation rate of hESCs was measured by 5-bromo-2'-deoxyuridine (BrdU) assay and telomerase activity. To evaluate differentiation potentials for hESCs, expression levels of undifferentiated and/or differentiated hESCs markers were examined by FACS, RT-PCR and immunostaining. Population-doubling time of early passage hESCs was longer than those of middle or late passage. Proliferative ability of $h E S C s$ was accelerated depending on
\end{abstract}

culture periods. Cellular morphologies and the expression level of each three germ layer markers were obviously different from each passage of reattached embryoid bodies (EBs) after spontaneous differentiation. Differentiated cells of late passage expressed higher levels of undifferentiated markers such as Oct4 and SSEA4 than those of early and middle passage. But differentiated cells of early and middle passage expressed higher level of differentiated state markers, Nestin (ectoderm), Brachyury (mesoderm), HNF3 $\beta$ (endoderm). From these results, it can be inferred that hESCs show higher proliferative abilities and reduced differentiation potentials as the passage number increased. Therefore, we conclude that early passage hESCs could be more suitable than middle and late passage $\mathrm{hESC}$ in differentiation studies.

Keywords: cell differentiation; cell proliferation; embryonic stem cells; humans

\section{Introduction}

Embryonic stem cells (ESCs), derived from inner cell mass (ICM) of preimplantation embryos, can proliferate indefinitely in undifferentiated state and maintain a normal karyotype in vitro (Evans and Kaufman, 1981). Also, ESCs have ability to differentiate into any cell type under proper culture conditions (Dushnik-Levinson and Benvensity, 1995). Since the first report on derivation of hESCs was issued by Thomson et al. (1998), many studies have been performed to identify genes and factors related to early human development and differentiation in order to use hESCs as a cell source for cell-based therapy. However, the use of hESCs for cell-based therapy requires detailed protocols for their differentiation into given cell types and subsequent isolation. Also, issues such as immune rejection and the transmission of unknown pathogens from mouse feeder cell must be solved before human transplantation is undertaken.

The stabilities of hESCs cultured in vitro seem to depend on the culture environment (Draper et al., 2004). When undifferentiated hESCs were cultured for prolonged period, some population of undifferentiated hESCs might be altered. These cells are morphologically undistinguishable under the microscope regardless of the expansion method 
(Oh et al., 2005b), although they show enhanced growth and proliferation. In addition, these altered cells may become the major population in longterm culture of hESCs in vitro (Smith, 2001). In mouse ESCs, alterations in pluripotency can occur because of chromosomal abnormalities during long-term culture (Longo et al., 1997). In hESCs, Draper et al. (2004) reported chromosomal abnormalities with an addition in chromosome $17 q$ and 12 in long-term culture of undifferentiated hESCs.

It is well known that undifferentiated hESCs can maintain their pluripotent characteristics stably in spite of an extended period of in vitro cultivation. However, we have been encountered changes in proliferative ability and differentiation potential during extended culture of hESCs. We observed that proliferative ability of hESCs was accelerated and differentiation potential was reduced as the number of passage increased. In addition, patterns of human embryoid body (EB) formation were differed from each group. As the number of passage increased, the ratio of simple EB formation was increased whereas cystic EB formation decreased.

To analyze the alterations in stability of hESCs, we examined population-doubling time, proliferation rate and change of expression level of specific markers related to undifferentiated and differentiated states according to extended culture periods.

\section{Materials and Methods}

\section{Materials}

A human embryonic stem cell line, SNUhES3, was used in this study. SNUhES3 has a normal karyotype (46, XY) and expresses undifferentiated hESC specific markers such as alkaline phosphatase, Oct4, SSEA3, SSEA4, Tra-1-60 and Tra-1-81 (Oh et al., 2005a). We defined passages up to 40 as early passage, up to 90 as middle passage, and up to 130 as late passage.

\section{Culture and spontaneous differentiation of hESCs}

Undifferentiated hESCs were maintained on mitomycin C (Sigma, St. Louis, MO) treated STO (ATCC, Manassas, VA) feeder layer. Undifferentiated hESC colonies were dissected using a glass pipette and replated onto a fresh feeder layer every 7 days. DMEM/F12 (Invitrogen, Carlsbad, CA), supplemented with $20 \%$ knockout serum replacement (Invitrogen), 1\% nonessential amino acids (Invitrogen), $0.1 \mathrm{mM} \beta$-mercaptoethanol (Sigma), $0.4 \mathrm{ng} / \mathrm{ml} \mathrm{bFGF}$ (Invitrogen), $50 \mathrm{U} / \mathrm{ml}$ penicillin (Invitrogen) and $50 \mu \mathrm{g} / \mathrm{ml}$ streptomycin (Invitrogen), was used as hESC culture medium.
For spontaneous differentiation, undifferentiated hESC colonies were cultured for 6 days and then treated with $2 \mathrm{mg} / \mathrm{ml}$ collagenase type IV (Invitrogen) for $30 \mathrm{~min}$ at $37^{\circ} \mathrm{C}$ to detach the colonies from the dish bottom. Detached hESC colonies were transferred to bacterial dishes and cultured for 2 weeks in suspension for the formation and spontaneous differentiation of EBs. hESC culture medium without bFGF was used as EB culture medium. After 2 weeks, EBs were reattached onto $0.1 \%$ gelatin-coated culture dish and cultured for 7 days for further differentiation.

\section{Measurement of population-doubling time}

We defined the population-doubling time as the time required for a colony area to expand two-fold. Population-doubling time was measured daily from 2 days after plating of undifferentiated hESCs to 6 days and the approximate area of hESC colonies was calculated by using the area equation of ellipse [S $=\pi \times$ a (horizontal diameter) $\times b$ (longitudinal diameter) / 4] (Reubinoff et al., 2001). The area of ten colonies was measured in early, middle and late passage, respectively. Population-doubling time was analyzed by regression curve analysis using an exponential series function.

\section{Karyotyping}

For mitotic preparations, hESCs were cultured in hESC medium supplemented with $0.1 \mathrm{mg} / \mathrm{ml}$ colcemid (Invitrogen) for up to $4 \mathrm{~h}$. The cells were harvested and fixed according to the GTG banding method for standard chromosome analysis (Seol et al., 2007). More than twenty of metaphases were karyotyped in the hESCs using chromosome imaging analyzer system (Chips, GenDix, Korea).

\section{BrdU incorporation assay}

Undifferentiated hESCs of early, middle and late passage were labeled with $10 \mu \mathrm{M}$ 5-bromo-2deoxyuridine (BrdU, Roche Diagnostics, Germany) for $24 \mathrm{~h}$ to assess mitotic activity. After washing with PBS, hESC colonies were detached from the dish bottom using $0.5 \mathrm{mg} / \mathrm{ml}$ dispase (Invitrogen). Detached colonies were then incubated with $0.25 \%$ trypsin-EDTA (Invitrogen) for $7 \mathrm{~min}$ and dissociated into single cells by repetitive pipetting. Single cells were fixed with $70 \% \mathrm{EtOH}$ for $30 \mathrm{~min}$ at room temperature (RT). After washing with PBS, cells were denatured with $4 \mathrm{M} \mathrm{HCl}$ for $10 \mathrm{~min}$ at RT. Then cells were repeatedly washed with PBS until the $\mathrm{pH}$ reached 6.5 or above, and incubated with blocking solution for $30 \mathrm{~min}$ at RT to prevent 
nonspecific reactions. Mouse anti-BrdU antibody (Chemicon, Temecula, CA) was added and incubated for $45 \mathrm{~min}$ at $37^{\circ} \mathrm{C}$. After washing three times with PBS, Alexa Fluor 488-labeled donkey antimouse IgG antibody (Molecular Probes, Eugene, OR) was added and incubated for $45 \mathrm{~min}$ at $37^{\circ} \mathrm{C}$ and then cells were washed three times with PBS. Propidium lodide (PI; Sigma) was added and incubated for $30 \mathrm{~min}$ at RT to distinguish live cells from dead cells. Cells were analyzed using a BD FACS Calibur $^{\mathrm{TM}}$ (BD Sciences, San Jose, CA).

\section{Quantitative real-time PCR}

Total RNA was extracted using RNeasy mini kit (Qiagen, Valencia, CA) according to manufacturer's instructions. cDNA was synthesized from 1 $\mu \mathrm{g}$ of total RNA using the Superscript II first-strand synthesis system (Invitrogen). Quantitative PCR was carried out in Rotor-Gene 3000 (Corbett Life Science, Sydney, Australia) using the QuantiTect SYBR green PCR kit (Qiagen). Primers used for the reactions are listed in Table 1 . The amplification program included an initial step at $95^{\circ} \mathrm{C}$ for $15 \mathrm{~min}$, followed by 45 cycles of denaturation at $95^{\circ} \mathrm{C}$ for $15 \mathrm{~s}$, annealing at $58^{\circ} \mathrm{C}$ for $20 \mathrm{~s}$ and extension at $72^{\circ} \mathrm{C}$ for $30 \mathrm{~s}$. All reactions were run in triplicate. The $\mathrm{C}_{\mathrm{T}}$ was defined as the cycle at which a statistically significant increase in the magnitude of the signal generated by the PCR reaction was first detected. $C_{T}$ was calculated under default settings for the Rotor-gene 6 program (Corbett Life Science). Relative gene expression was normalized to GAPDH expression.

\section{TRAP assay}

Telomerase activity was detected using TRAP EZE $_{\text {E }}$ telomerase detection kit (Chemicon) according to manufacturer's instruction. Briefly, purified protein concentrations were determined by Bradford assay kit (Bio Rad, Hercules, CA) using BSA as a standard. To make heat-inactivated control, proteins were heat-inactivated at $85^{\circ} \mathrm{C}$ for $10 \mathrm{~min}$. PCR was performed as 2-step PCR at $94^{\circ} \mathrm{C}$ for 30 $s$ and at $59^{\circ} \mathrm{C}$ for $30 \mathrm{~s}$ for 30 cycles. PCR products were analyzed on a $12.5 \%$ nondenaturing polyacrylamide gel and stained with SYBR green I (Sigma).

\section{FACS analysis}

Undifferentiated hESC colonies were detached using $2 \mathrm{mg} / \mathrm{ml}$ collagenase type IV. After washing three times with PBS, $0.25 \%$ trypsin-EDTA was treated to dissociate them into single cells. After washing twice with PBS, blocking solution includeing $0.05 \%$ Triton $\times 100$ (Sigma) was treated. Rabbit anti-human Oct4 (Santa Cruz Biotechnology, Santa Cruz, CA) and mouse anti-human SSEA4 antibodies (Chemicon) were used as primary antibodies. Secondary antibodies, Alexa Fluor 488-labeled donkey anti-mouse IgG and 594labeled donkey anti-rabbit IgG (Molecular probes), were treated for $30 \mathrm{~min}$ and then washed three times with PBS. Cells were analyzed using BD FACS Calibur $^{\mathrm{TM}}$ (BD Sciences).

\section{RT-PCR}

Total RNA was extracted using RNeasy mini kit (Qiagen) according to manufacturer's instructions. cDNAs were synthesized from $0.5 \mu \mathrm{g}$ of RNA using first strand cDNA synthesis kit for RT-PCR (Roche Diagnostics). PCR was performed with HiPi PCR PreMix (Elpis Biotech, Daejeon, Korea) with each primer. Each primer sequence, product size, annealing temperature and cycle numbers used for the reaction are listed in Table 1 . PCR products were analyzed in $2 \%$ agarose gel.

Table 1. Primer sequences and conditions used in this study.

\begin{tabular}{|c|c|c|c|c|c|c|}
\hline \multirow{2}{*}{ PCR } & \multirow{2}{*}{ Gene } & \multicolumn{2}{|c|}{ Sequences } & \multirow{2}{*}{$\begin{array}{l}\text { Product } \\
\text { size (bp) }\end{array}$} & \multirow{2}{*}{$\begin{array}{l}\text { Annealing } \\
\text { temp }\left({ }^{\circ} \mathrm{C}\right)\end{array}$} & \multirow{2}{*}{$\begin{array}{l}\text { No. of } \\
\text { cycles }\end{array}$} \\
\hline & & Forward & Reverse & & & \\
\hline \multirow{6}{*}{ RT-PCR } & GAPDH & AGCCACATCGCTCAGACACC & GTACTCAGCGGCCAGCATCG & 302 & 60 & 28 \\
\hline & Oct4 & GAGAACAATGAGAACCTTCAGGA & TTCTGGCGCCGGTTACAGAACCA & 219 & 60 & 27 \\
\hline & Nanog & TGCCTCACACGGAGACTGTC & TGCTATTCTTCGGCCAGTTG & 380 & 60 & 28 \\
\hline & Nestin & CAGCTGGCGCACCTCAAGATG & AGGGAAGTTGGGCTCAGGACTGG & 209 & 60 & 28 \\
\hline & Brachyury & ATCGTGGACAGCCAGTACGA & GCCAACTGCATCATCTCCAC & 339 & 55 & 38 \\
\hline & HNF3 $\beta$ & CTACGCCAACATGAACTCCA & GAGGTCCATGATCCACTGGT & 199 & 58 & 38 \\
\hline \multirow{3}{*}{$\begin{array}{l}\text { Quantitative } \\
\text { real-time } \\
\text { PCR }\end{array}$} & GAPDH & GGCGTTCTCTTTGGAAAGGTGTTC & GTACTCAGCGGCCAGCATCG & & & \\
\hline & CyclinD1 & GATCAAGTGTGACCCGGACT & тсСтсстсСтсттсстсСтс & & & \\
\hline & CyclinD2 & GTCTCAAAGCTTGCCAGGAG & ATATCCCGCACGTCTGTAGG & & & \\
\hline
\end{tabular}




\section{Immunostaining}

hESCs were washed with PBS and fixed sequentially with $70 \%, 80 \%, 90 \%$ and absolute $\mathrm{EtOH}$ for $5 \mathrm{~min}$ at RT. Perforation solution was treated for $10 \mathrm{~min}$ at RT and washed with PBS. To block nonspecific reaction, cells were incubated with $3 \%$ BSA solution for overnight at $4^{\circ} \mathrm{C}$. After washing with PBS, rabbit anti-human Oct4 (Santa Cruz), mouse anti-human SSEA4 (Chemicon), mouse anti-human Nestin (R\&D Systems, Minneapolis, $\mathrm{MN}$ ), rabbit anti-human Brachyury (Santa Cruz) and goat anti-human HNF3 $\beta$ (Santa Cruz) antibodies were treated for $1 \mathrm{~h}$ at RT. After washing with PBS for three times, Alexa Fluor 594-labeled donkey anti-goat IgG, Alexa Fluor 594-labeled donkey anti-rabbit IgG and Alexa Fluor 488-labeled donkey anti-mouse IgG antibodies were treated and then washed with PBS for three times. Cells were analyzed using a confocal microscope (Bio Rad).

\section{Results}

\section{Changes in proliferation rate of hESCs according to culture period}

To evaluate changes in proliferation rate according to culture period, we calculated population-doubling time indirectly by measuring colony area. Ten colonies from each group were evaluated from day 2 to 6 after subculturing. We measured the length of the major and minor axis of the hESC colonies, calculated hESC colony area using these parameters and determined population-doubling time by regression curve analysis. In the early passage, population-doubling time was $37.6 \mathrm{~h}$, whereas population-doubling time of middle or late passage was about $30 \mathrm{~h}$. These results demonstrate that the population-doubling time of hESC colonies differed according to culture period and reduced as passage number was increased (Figure $1 \mathrm{~A}$ ). To examine whether the altered characteristics of

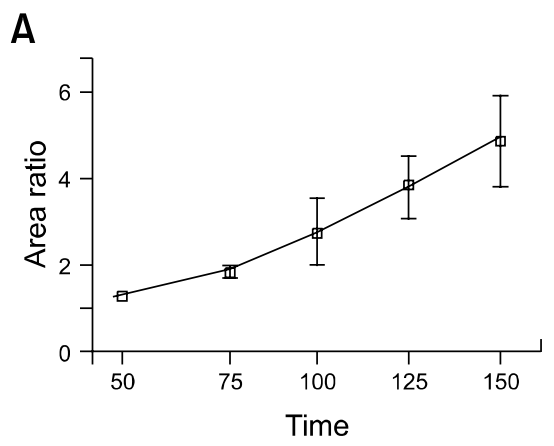

Early passage

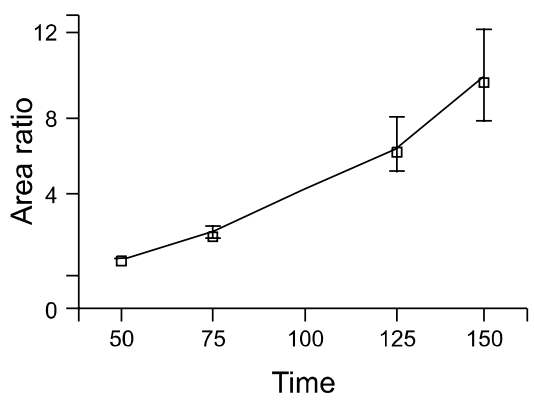

Middle passage

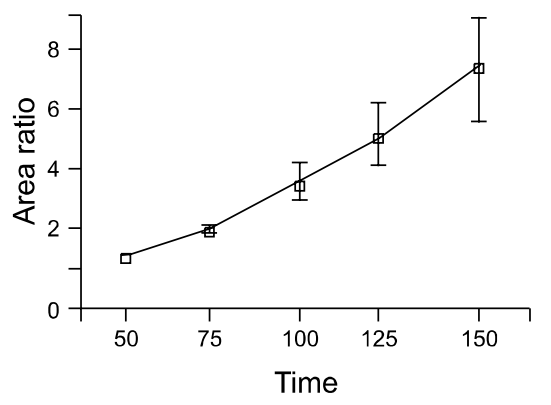

Late passage

\begin{tabular}{l|ccc}
\hline & Expected equation & R-square & Doubling time \\
\hline Early passage & $\mathrm{A}=0.405 \mathrm{e}^{\wedge} 0.0184 \mathrm{t}$ & 0.91344 & $37.61 \mathrm{~h}$ \\
Middle passage & $\mathrm{A}=0.367 \mathrm{e}^{\wedge} 0.0233 \mathrm{t}$ & 0.95657 & $29.81 \mathrm{~h}$ \\
Late passage & $\mathrm{A}=0.304 \mathrm{e}^{\wedge} 0.0226 \mathrm{t}$ & 0.91542 & $30.67 \mathrm{~h}$ \\
\hline
\end{tabular}

B

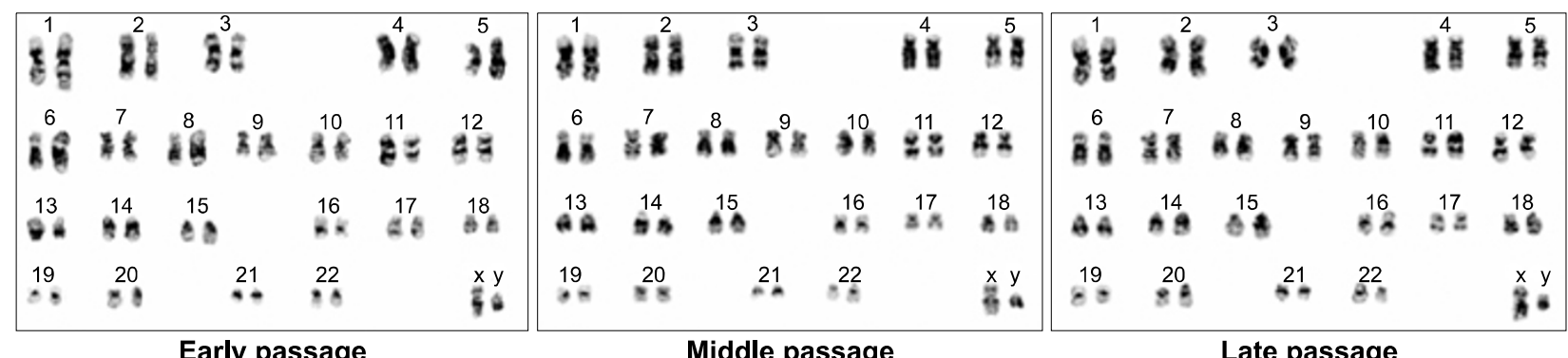

Figure 1. Population-doubling time and karyotype of human embryonic stem cells (hESCs). (A) The area ratio was calculated by the area of day2 hESC colonies. (B) Karyotype of hESCs was analyzed by GTG banding method for standard chromosome analysis. 
hESCs caused by chromosomal abnormalities, we performed cytogenetic analysis in each passage group. All hESCs maintained normal karyotype $(46, X Y)$ independent of culture period (Figure 1B).

\section{Differences of BrdU incorporation, CyclinD expression and telomerase activity of hESCs}

To assess the fraction of cells in $S$ phase, we performed BrdU incorporation assay in early, middle and late passage of hESCs, respectively. Undifferentiated hESCs in each group were incubated with $10 \mu \mathrm{M}$ BrdU for 24 h. Propidium lodide $(\mathrm{Pl})$ was added to distinguish between live and dead cells from the population. Most cells were positive for BrdU and portion of the dead cells was under $10 \%$ (data not shown). In early passage, $62.1 \%$ of hESCs were positive for BrdU. In middle passage, $90.3 \%$ of hESCs were positive, and $94.6 \%$ were positive in late passage. All three hESC groups actively proliferated, but middle and late passage hESCs showed more active in proliferation than early passage $\mathrm{hESCs}$ (Figure $2 \mathrm{~A}$ ). We also measured the expressions of CyclinD1 and $D 2$ and all three groups highly expressed CyclinD1 and D2. Expression of CyclinD1 and D2

A

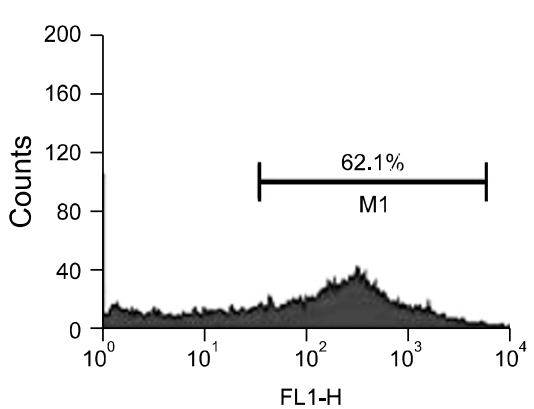

Early passage

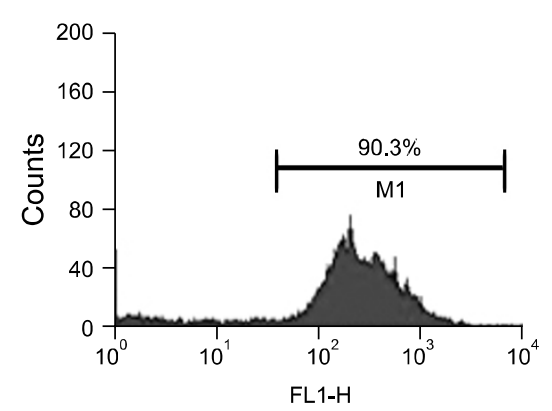

Middle passage

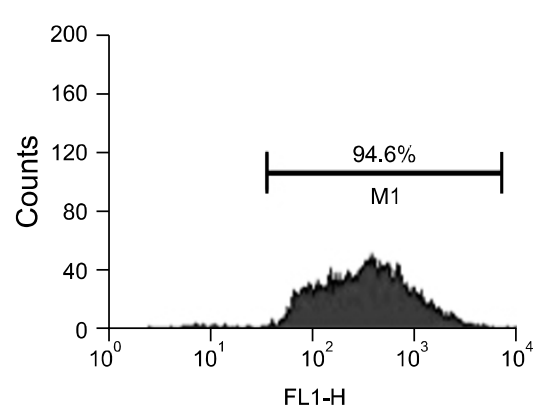

Late passage

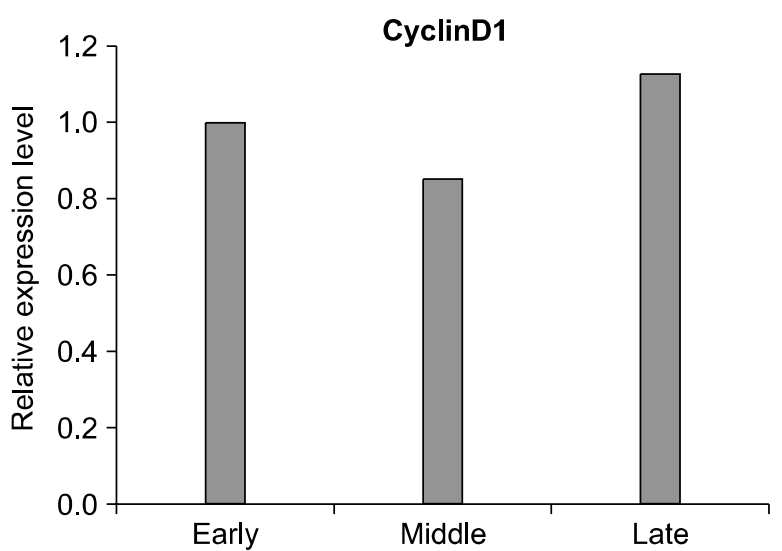

B

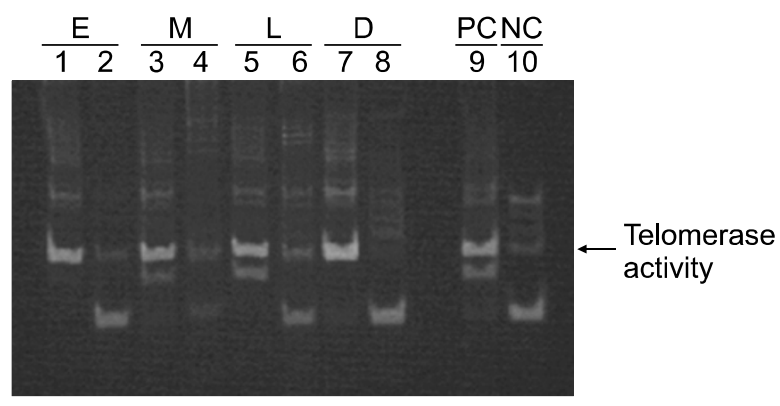

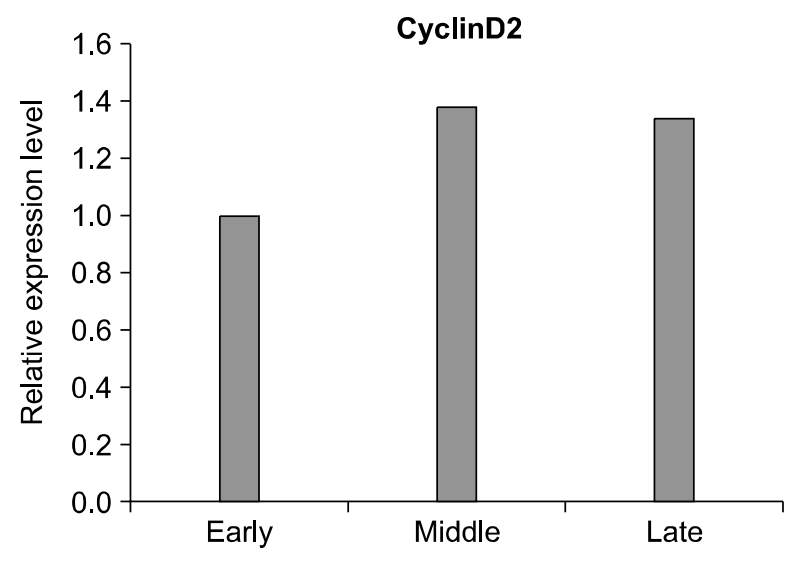

Figure 2. Differences of BrdU incorporation, CyclinD expression and telomerase activity in hESCs according to culture period. (A) BrdU incorporation assay of early, middle and late group in hESCs. Cells were treated with $10 \mu \mathrm{M}$ of BrdU for $24 \mathrm{~h}$. After incubation, samples were trypsinized to dissociate into single cells and labeled with BrdU antibody. Expressions of Cyclins were analyzed using quantitative real-time PCR. mRNA expression levels were normalized to GAPDH expression. Relative gene expression was determined by normalization to that of early passage group. (B) Telomerase activities in each group of hESCs. Telomerase activities of early, middle and late passaged hESCs were analyzed using TRAP assay. Heat-inactivated controls were heat-inactivated at $85^{\circ} \mathrm{C}$ and loaded beside each sample. $\mathrm{E}$, early passage; $\mathrm{M}$, middle passage; $L$, late passage; $D$, differentiated hESCs; lane 1, 3, 5, 7, untreated hESCs; lane 2, 4, 6, 8, heat-inactivated hESCs; PC, positive control provided by kit; $\mathrm{N}$, negative control. 
was slightly increased in late passage compared to early passage, however, the expression level of CyclinD2 in middle and late passage hESCs was not significantly different. These results demonstrate that proliferation rate of hESCs were accelerated as passage number increased. However, telomerase activities were high both in three groups of hESCs and differentiated hESCs (Figure 2B).

\section{Changes in differentiation potential of hESCs}

To compare differentiation potential according to extended culture period, we firstly evaluated the expression of undifferentiated state markers in each group using FACS, RT-PCR and immunostaining. FACS analysis results indicated that a high proportion of undifferentiated hESCs in all three groups commonly expressed undifferentiated state markers, Oct4 and SSEA4. All three groups expressed high levels of undifferentiated state markers, but differences in positive population between them were not apparent. Expression of Oct4 and SSEA4 were $98.52 \%$ and $98.76 \%$ in the early passage group, $88.79 \%$ and $98.44 \%$ in the middle passage group, $90.05 \%$ and $99.54 \%$ in the late passage group, respectively (Figure 3 ). Secondly, to evaluate the changes of differentiation potential during differentiation, each group of hESCs was spontaneously differentiated as follows: EBs were made from each passage of hESCs and reattached to induce further differentiation. EBs classified into simple and cystic forms by morphological appearance. All three groups of hESCs generated EB efficiently, although more cystic EBs were produced from early and middle passage hESCs than late passage, and the majority of EBs from late hESCs were simple EBs (Figure 4A-C). The expression of undifferentiated state markers Oct4 and Nanog was analyzed by RT-PCR. No significant differences in the expressions of Oct4 and Nanog were observed in all three groups of undifferentiated hESCs. In EBs and reattached cells, the expression levels of Oct4 and Nanog were higher in late passage than in the early and middle passage (Figure 5A). However, Oct4 and Nanog were all highly expressed in simple EBs, but their expression reduced in cystic EBs (Figure 5B). These mean that cystic EBs possessed more differentiated proportion of cells than simple EBs. Also, we demonstrate that Oct4 and Nanog are highly expressed in long-term cultured hESCs though the cells were differentiated spontaneously. These findings suggest that Oct4 and Nanog expression are maintained by a low incidence of

\section{Oct4}

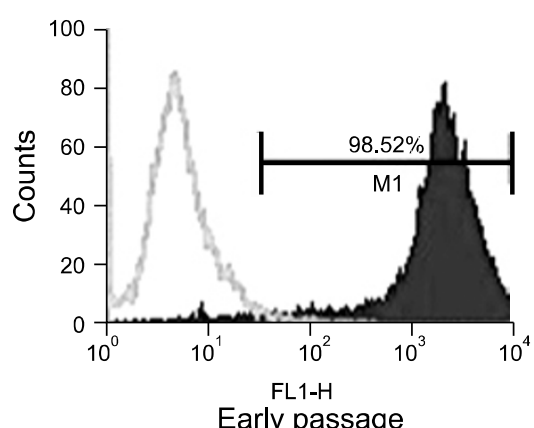

Early passage

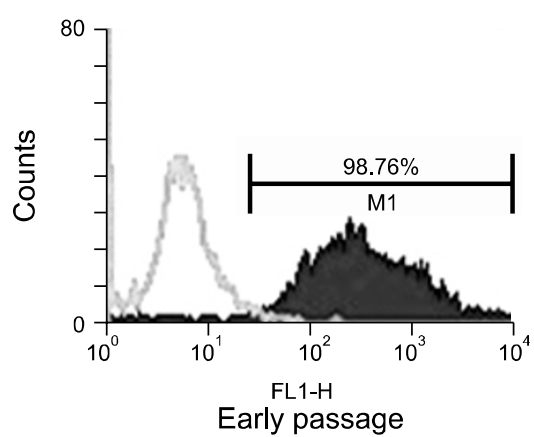

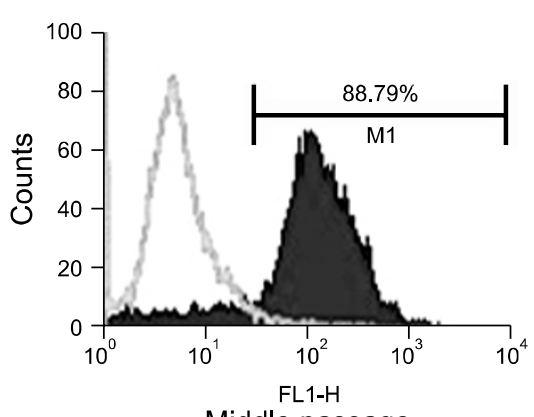

Middle passage

SSEA4

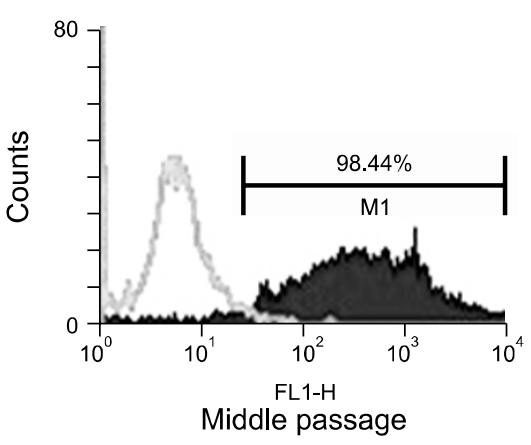

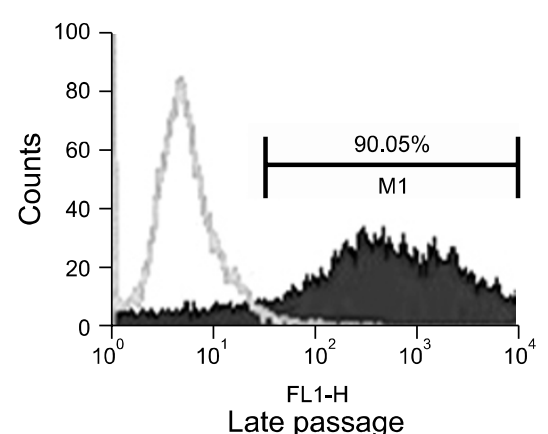

Late passage

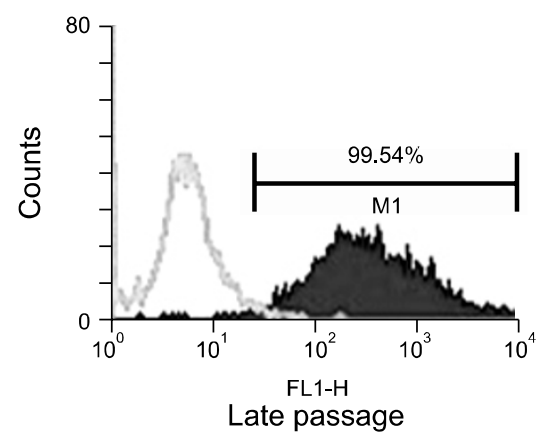

Figure 3. FACS analysis of undifferentiated markers, Oct4 and SSEA4, in the early, middle and late passage of undifferentiated hESCs. 

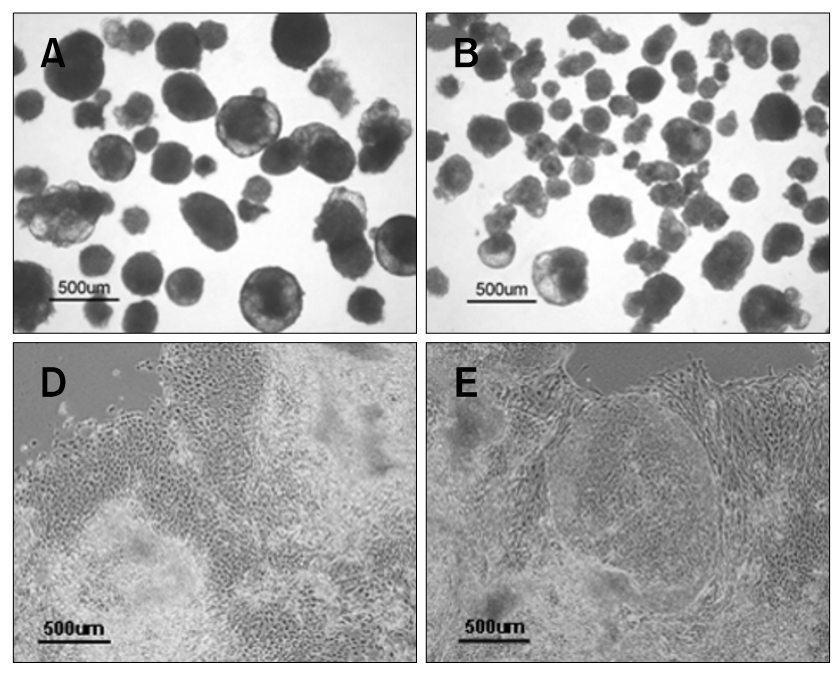

A

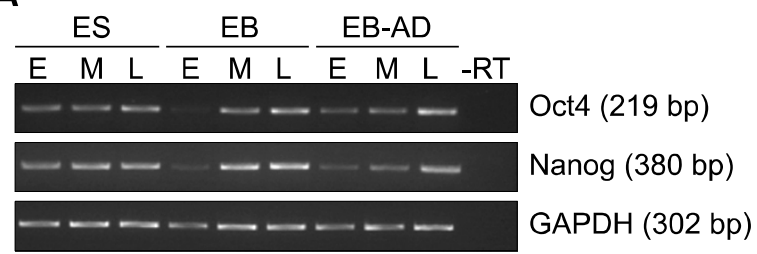

B

$\begin{array}{ll}\frac{\text { Early EBs }}{\text { Simple Cystic }} \frac{\text { Middle EBs Late EBs }}{\text { Simple Cystic } \frac{\text { Simple }}{\text { Sim }}} & \text { Oct4 (219 bp) } \\ - & \text { Nanog (380 bp) } \\ - & \text { GAPDH (302 bp) }\end{array}$

Figure 5. RT-PCR for the expressions of undifferentiated state markers from each group of hESCs and EBs. (A) Expression levels of Oct4 and Nanog in human embryonic stem cells (ES), embryoid bodies (EB) and reattached embryoid bodies (EB-AD). (B) Expression levels of Oct4 and Nanog in simple and cystic EBs from each passage group. $E$, early passage; $M$, middle passage; L, late passage; -RT, PCR without addition of reverse transcriptase.

spontaneous differentiation and that hESCs have a tendency to proliferate rather than differentiate on prolonged culture.

As the spontaneous differentiation progressed, in early group, reattached cells from EBs were differentiated into various cell types including polygonal cells (Figure 4D). Reattached cells from middle group contained a small proportion of undifferentiated cells (Figure 4E). However, reattached cells from the late group showed a higher proportion of undifferentiated cells than those from the early and middle groups (Figure 4F). To confirm these findings, we evaluated the expression of
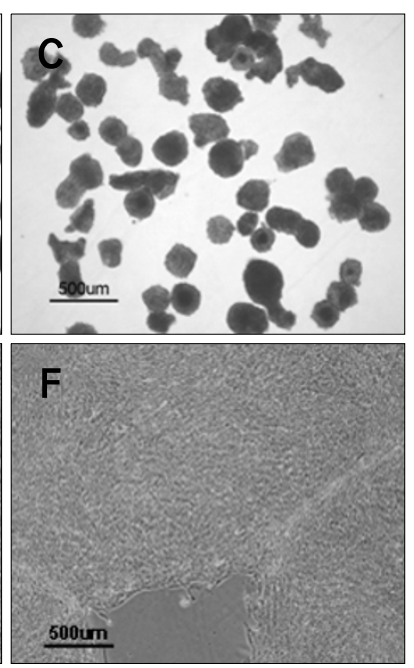

Figure 4. Morphologies of EBs and reattached cells of EBs from early $(A, D)$, middle $(B, E)$, late $(C, F)$ passage groups. Upper panel (A-C) showed EBs formed from each group and lower panel (D-F) showed reattached cells of EBs from each group.

Oct4 and SSEA4 as undifferentiated state markers by immunostaining. Reattached cells from the early, middle and late group of EBs still expressed Oct4 and SSEA4 in spontaneously differentiated cells, but their expression was significantly reduced in the early group, not in the late group (Figure 6).

In addition, we evaluated the expressions of Nestin, Brachyury, HNF3 $\beta$ as differentiated hESCs markers by immunostaining, which represent ectoderm, mesoderm, endoderm, respectively. All three germ layer markers were expressed in all passage groups. But the expressions were significantly reduced in late group (Figure 7A). Also RT-PCR analysis showed similar expression pattern to that of immunostaining (Figure 7B).

\section{Discussion}

Since the derivation of hESCs (Thomson et al., 1998; Reubinoff et al., 2000; Oh et al., 2005a), successful differentiation studies of hESCs into various cell types were reported (Rambhatla et al., 2003; Snir et al., 2003; Segev et al., 2004; Kang et al., 2006). Many studies continue on subjects as diverse as developmental regulation and determination of the characteristics and development of cell lines containing genetic modifications that are suitable for cell-based therapy (Gropp et al., 2003; Ma et al., 2003).

hESCs generally maintain their novel characteristics, such as expression of undifferentiated cell surface antigens, transcription factors and telomerase activity after continuous long-term culture or even after cryopreservation (Ha et al., 2005), but some alterations in characteristics, including proliferation rate and differentiation potential, are 


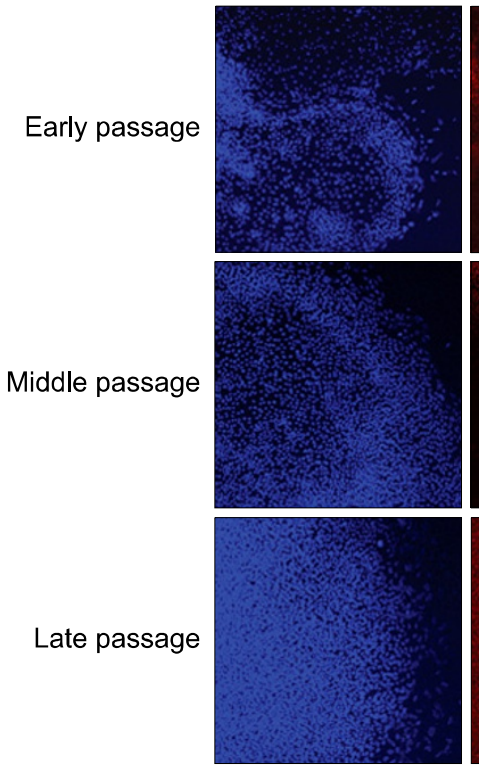

DAPI
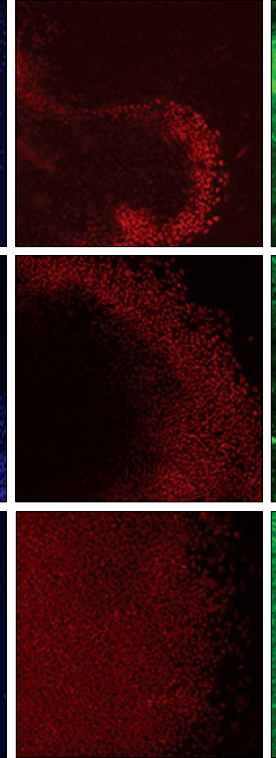

Oct4

A

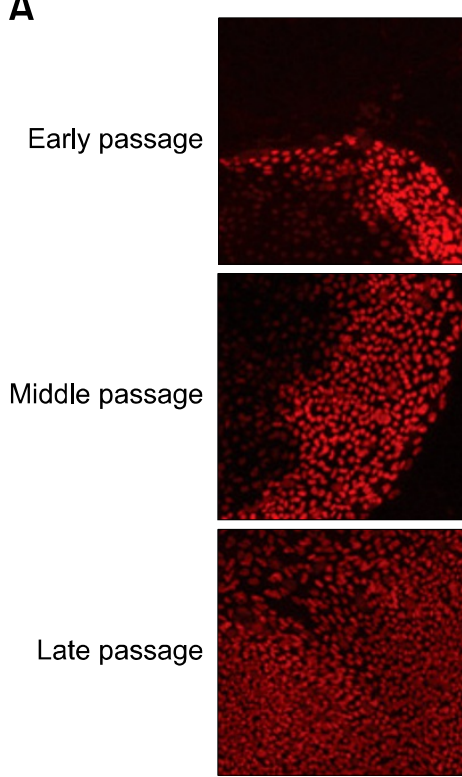

Oct4
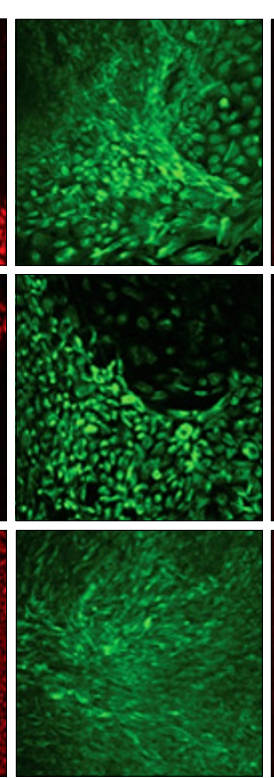

Nestin

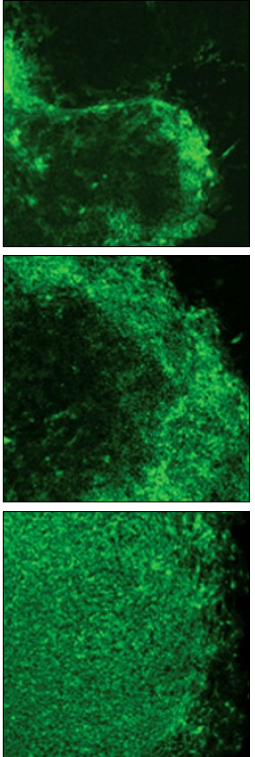

SSEA4
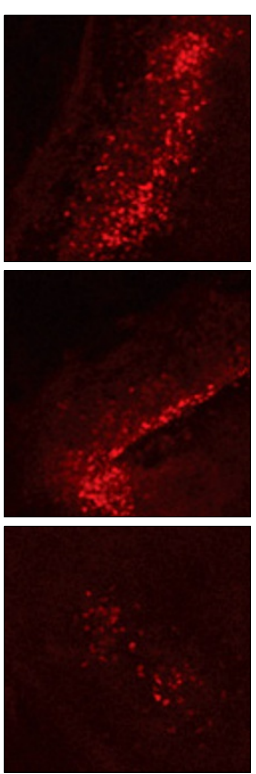

Brachyury

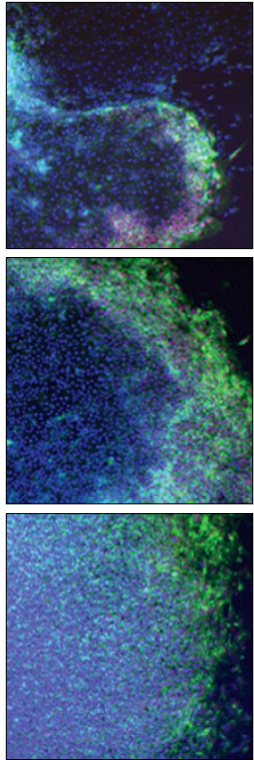

Merge
Figure 6. Immunostaining of undifferentiated state markers in reattached EBs from each group of hESCs. Expressions of Oct4 and SSEA4 in reattached EBs were observed by confocal microscope.

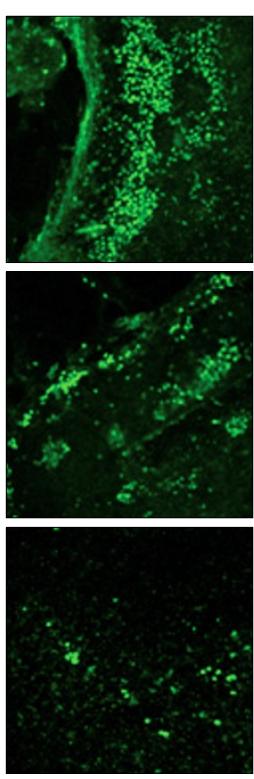

HNF3 $\beta$
Figure 7. Immunostaining and RTPCR of three germ layer markers in
B

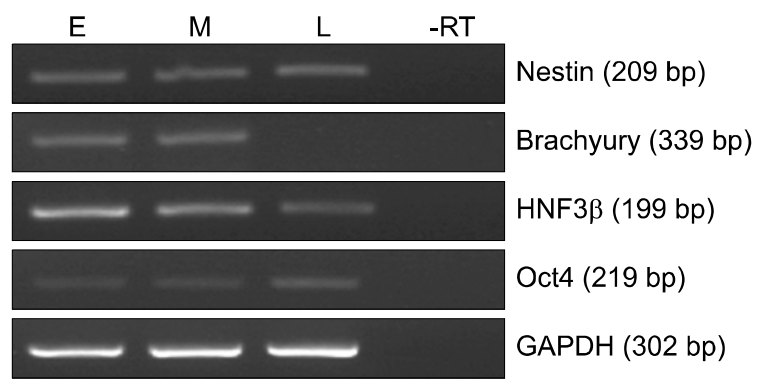
reattached EBs from each group of hESCs. (A) Expressions of Nestin, Brachyury, HNF3 $\beta$ in reattached EBs were observed by confocal microscope. (B) Expressions of Nestin, Brachyury, HNF3 $\beta$ in reattached EBs were analyzed by RT-PCR. Expression level of three germ layer markers were detected in reattached EBs from each group of hESCs. $E$, early passage; $M$, middle passage; $L$, late passage; -RT, PCR without addition of reverse transcriptase. 
appeared. In previous reports, researchers have demonstrated that maintaining pluripotent state in long-term culture may lead to alterations of stability of hESCs. Rosler et al. (2004) reported that a small proportion of long-term cultured cells could undergo genetic mutation and the mutated cells probably possessed enhanced growth rate or proliferative ability. Moreover, adaptation to culture conditions shortens population-doubling time of pluripotent stem cells and these cells also respond more flexibly to environmental stimuli or stress than less adapted cells. These kinds of changes may be lead into epigenetic alterations in the cells. Several studies are ongoing to evaluate the epigenetic status of various hESC lines maintained under different culture conditions (Maitra et al., 2005; Rugg-Gunn et al., 2005). Gene profiling analysis is commonly used to analyze the variety of established hESCs. Maitra et al. (2005) reported an alteration in the cellular DNA of late passage hESCs. Their findings suggest that hESCs may be sensitive to culture conditions for maintaining pluripotency and that hESCs are epigenetically regulated by environmental factors. Also, Draper et al. (2004) reported chromosomal abnormality, namely additions to chromosome $17 q$ and 12 in long-term cultured hESCs. Chromosome $17 q$ is known to contain genes related to the regulation of apoptosis and differentiation (Burdon et al., 2002; Chiou et al., 2003) and chromosome 12 contains genes related to the regulation of pluripotency in human embryonic carcinoma cells (Mostert et al., 1998). Based on these reports, they concluded that the addition of chromosome $17 q$ and 12 could be explained the adaptation, i.e. increased proliferative ability of hESCs according to culture environment (Draper et al., 2004). Recently, Baker et al. (2007) reported that genetic change of hESCs increased the capacity of cells to proliferate. They concluded that changes in hESCs in culture reflect tumorigenic events that occur in vivo, particulary in testicular germ cell tumors.

In our laboratory, we observed changes of characteristics in long-term cultured hESCs. These alterations included changes in population-doubling time, lower ratio of cystic EB formation, and reduced differentiation potential. To analyze the changes in the characteristics of long-term cultured hESCs, we evaluated different passage of cells and found that characteristics of hESCs were dependent on in vitro culture period. In the present study, we evaluated the changes in populationdoubling time and the expression patterns of undifferentiated state markers in hESCs, EBs and differentiated cells according to in vitro culture period. Also, expressions of three germ layer markers were evaluated with respect to differentiation potential.

We measured population-doubling time and found that the mean value was $37.6 \mathrm{~h}$ in the early group and $30 \mathrm{~h}$ in the late group, which was about $8 \mathrm{~h}$ shorter than in the early group. This result indicates that characteristics of hESCs were possibly changed depending on culture period and that late passage hESCs appeared to be grown faster and adapted stably to culture environment than the early group.

It was reported that pluripotent cells including mESCs (Savatier et al., 1994; Stead et al., 2002), and murine embryonal germ cells (Resnick et al., 1992) have a cell cycle structure lacking fully formed G1 and G2 gap phases. Recently, Becker et al. (2006) reported that hESCs showed unique cell cycle structure, which was shortened $\mathrm{G} 1$ cell cycle phase and most of cells were in $S$ phase. In this study, we found that most of hESCs in each group were positive for BrdU and these results indicated that most of hESCs were in S phase. In early passage, $62.1 \%$ of hESCs were positive. In middle and late passage, $90.3 \%$ and $94.6 \%$ were positive. We also evaluated expression of CyclinD1 and $D 2$ in each passage group. D type cyclins are known to promote $\mathrm{G} 1$ phase cell cycle regulation (Wianny et al., 1998, White and Dalton, 2005) and highly expressed in hESCs (Becker et al., 2006). CyclinDs were highly expressed in all three passage groups. From these results, long-term cultured cells have more proliferative abilities.

Telomerase is a ribonucleoprotein that adds telomere repeats to chromosome ends and is responsible for maintaining telomere length, which plays an important role in the replicative life span of cells (Harley, 1991). hESCs generally highly express human telomere reverse transcriptase (hTERT) activity (Thomson et al., 1998; Oh et al., 2005a). In the present study, all of three groups of hESCs expressed high levels of telomerase activity, and this activity was not reduced in differentiated cells. These results explain that all three groups have different proliferative activity, however, their telomerase activity was not affected by increasing passage number.

All from early, middle and late passage of hESCs showed typical morphology of undifferentiated hESC colonies regardless of culture period. FACS analysis showed that the specific transcription factor Oct4 and the cell surface antigen SSEA4 were highly expressed in all three groups. In addition, different characteristics were observed in the EBs formed from the three groups. Conley et al. (2004) reported that mouse ESCs and hESCs spontaneously differentiated into sphere termed 
EBs when cultured in suspension. EBs are classified into two types. Simple EBs is generally formed after 2-3 days and endoderm is formed outer layer of EBs. Cystic EBs are more differentiated structures bearing central cavity and double layered structure with an inner ectodermal layer and outer layer of endoderm enclosing cavity (Keller, 1995).

In this study, the early and middle passage groups formed simple and cystic EBs after suspension culture, whereas cystic EBs were rarely observed in the late passage group. RT-PCR results demonstrated that the expressions of Oct4 and Nanog in cystic EBs were low and slightly higher in those of middle passage group than in early passage group. However, the expressions of Oct4 and Nanog in simple EBs were equivalent in all three groups. This indicates that as passage number increased, the differentiation potential of hESCs was decreased. Immunostaining results showed that Oct4 and SSEA4 expression was significantly higher in the differentiated cells (i.e. EB reattached cells) produced from the hESCs of late passage group than those of early and middle passage group, although three germ layer markers, Nestin (ectoderm), Brachyury (mesoderm) and HNF3 $\beta$ (endoderm), were expressed in all three groups. However, the expression levels of three germ layer markers were significantly reduced in late passage. Ko et al. (2007) also reported that the degree of hESC differentiation varied depending on the passage number of undifferentiated hESCs in HSF-6 hES cell line. These results indicated that although long-term cultured hESCs retained somewhat their pluripotency, they also possessed a tendency to maintain an undifferentiated state rather than to differentiate.

In conclusion, the present study demonstrates that long-term culture in vitro alters the proliferative ability and differentiation potential of hESCs and analyzed the alterations in hESCs of early, middle and late passage. It was found that hESCs have a tendency to a higher proliferative ability and a reduced differentiation potential on increasing the passage number. These results show that hESCs are sensitive to culture environment and characteristics may be altered by extended culture. Therefore, we suggest that short-term cultured hESCs are more appropriate for various researches of hESCs.

\section{Acknowledgement}

This research was supported by grants (SC 2040 and 1020) from Stem Cell Research Center of the $21^{\text {st }}$ Century Frontier Research Program funded by the Ministry of Science and Technology, Korea.

\section{References}

Baker DE, Harrison NJ, Maltby E, Smith K, Moore HD, Shaw PJ, Heath PR, Holden H, Andrews PW. Adaptation to culture of human embryonic stem cells and oncogenesis in vivo. Nat Biotechnol 2007;25:207-15

Becker KA, Ghule PN, Therrien JA, Lian JB, Stein KL, van Wijnen AJ, Stein GS. Self-renewal of human embryonic stem cells is supported by a shortened G1 cell cycle phase. J Cell Physiol 2006;209:883-93

Burdon T, Smith A, Savatier P. Signaling, cell cycle and pluripotency in embryonic stem cells. Trends Cell Biol 2002;12:432-8

Chiou SK, Jones MK, Tarnawski AS. Surviving an antiapoptosis protein: its biological roles and implications for cancer and beyond. Med Sci Monit 2003;9:PI25-9

Conley BJ, Young JC, Trounson AO, Mollard R. Dervation, propagation and differentiation of human embryonic stem cells. Int J Biochem Cell Biol 2004;36:555-67

Draper JS, Smith K, Gokhale P, Moore HD, Maltby E, Johnson J, Meisner L, Zwaka TP, Thomson J, Andrews PW. Recurrent gain of chromosomes $17 q$ and 12 in cultured human embryonic stem cells. Nat Biotechnol 2004;22:53-4

Dushnik-Levinson M, Benvenisty N. Embryogenesis in vitro: study of differentiation of embryonic stem cells. Biol Neonate 1995;67:77-83

Evans MJ, Kaufman MH. Establishment in culture of pluripotential cells from mouse embryos. Nature 1981;292: 154-6

Gropp M, Itsykson P, Singer O, Ben-Hur T, Reinhartz E, Galun E, Reubinoff BE. Stable genetic modification of human embryonic stem cells by lentiviral vectors. Mol Ther 2003; 7:281-7

Ha SY, Jee BC, Suh CS, Kim HS, Oh SK, Kim SH, Moon SY. Cryopreservation of human embryonic stem cells without the use of a programmable freezer. Human Reproduction 2005;20:1779-85

Harley CB. Telomere loss: mitotic clock or genetic time bomb? Mutat Res 1991;256:271-82

Kang SM, Cho MS, Seo H, Yoon CJ, Oh SK, Choi YM, Kim DW. Efficient induction of oligodendrocytes from human embryonic stem cells. Stem Cells 2006;52:419-24

Keller GM. In vitro differentiation of embryonic stem cells. Curr Opin Cell Biol 1995;7:862-9

Ko JY, Park CH, Koh HC, Cho YH, Kyhm JH, Lim YS, Lee SH. Human embryonic stem cell-derived neural precursors as a continuous, stable, and on-demand source of human dopamine neurons. J Neurochem 2007;103:1417-29

Longo L, Bygrave A, Grosveld FG, Pandolfi PP. The chromosome make-up of mouse embryonic stem cells is predictive of somatic and germ cell chimaerism. Transgenic Res 1997;6:321-8

Ma Y, Ramezani A, Lewis R, Hawley RG, Thomson JA. High-level sustained transgene expression in human embryonic stem cells using lentiviral vectors. Stem Cells 


\section{3;21:111-7}

Maitra A, Arking DE, Shivapurkar N, Ikeda M, Stastny V, Kassauei K, Sui G, Cutler DJ, Liu Y, Brimble SN, Noaksson K, Hyllner J, Schulz TC, Zeng X, Freed WJ, Crook J, Abraham S, Colman A, Sartipy P, Matsui S, Carpenter M, Gazdar AF, Rao M, Chakravarti A. Genomic alterations in cultured human embryonic stem cells. Nature Genetics 2005;37: 1099-103

Mostert MC, Verkerk AJ, van de Pol M, Heighway J, Marynen P, Rosenberg C, van Kessel AG, van Echten J, de Jong B, Oosterhuis JW, Looijenga LH. Identification of the critical region of $12 p$ over-representation in testicular germ cell tumors of adolescents and adults. Oncogene 1998;16: 2617-27

Oh SK, Kim HS, Ahn HJ, Seol HW, Kim YY, Park YB, Yoon CJ, Kim DW, Kim SH, Moon SY. Derivation and characterization of new human embryonic stem cell lines, SNUhES1, SNUhES2, and SNUhES3. Stem Cells 2005a; 23:211-9

Oh SK, Kim HS, Park YB, Seol HW, Kim YY, Cho MS, Ku SY, Choi YM, Kim DW, Moon SY. Methods for expansion of human embryonic stem cells. Stem Cells 2005b;23:605-9

Rambhatla L, Chiu CP, Kundu P, Peng Y, Carpenter MK. Generation of hepatocyte-like cells from human embryonic stem cells. Cell Transplant 2003;12:1-11

Resnick JL, Bixler LS, Cheng L, Donovan PJ. Long-term proliferation of mouse primordial germ cells in culture. Nature 1992;10:87-95

Reubinoff BE, Pera MF, Fong CY, Trounson A, Bongso A. Embryonic stem cell lines from human blastocysts: somatic differentiation in vitro. Nat Biotechnol 2000;18:399-404

Reubinoff BE, Pera MF, Vajta G, Trounson AO. Effective cryopreservation of human embryonic stem cells by the open pulled straw vitrification method. Human Reproduction 2001;16:2187-94

Rosler ES, Fisk G, Ares X, Irving J, Miura T, Rao MS, Carpenter MK. Long-term culture of human embryonic stem cells in feeder-free conditions. Developmental Dynamics

\section{4;229:259-74}

Rugg-Gunn PJ, Ferguson-Smith AC, Pedersen RA. Epigenetic status of human embryonic stem cells. Nat Genet 2005;37:585-7

Savatier P, Huang S, Szekely L, Wiman KG, Samarut J. Contrasting patterns of retinoblastoma protein expression in mouse embryonic stem cells and embryonic fibroblasts. Oncogene 1994;9:809-18

Segev H, Fishman B, Ziskind A, Shulman M, Itskovitz-Eldor $J$. Differentiation of human embryonic stem cells into insulin-producing clusters. Stem Cells 2004;22:265-74

Seol HW, Oh SK, Kim HS, Yim MY, Baek JA, Suh J, Moon SY, Choi YM. A study of optimal methods for chromosome analysis of human embryonic stem cells. J Reproductive Medicine and Population 2007;20:86-93

Smith AG. Embryo-derived stem cells: of mice and men. Annu Rev Cell Dev Biol 2001;17:435-62

Snir M, Kehat I, Gepstein A, Coleman R, Itskovitz-Eldor J, Livne E, Gepstein L. Assessment of the ultrastructural and proliferative properties of human embryonic stem cellderived cardiomyocytes. Am J Physiol Heart Circ Physiol 2003;285:H2355-63

Stead E, White J, Faast R, Conn S, Goldstone S, Rathjen J, DhingraU, Rathjen P, Walker D, Dalton S. Pluripotent cell division cycles are driven by ectopic Cdk2, cyclin A/E and E2F activities. Oncogene 2002;21:8320-33

Thomson JA, Itskovitz-Eldor J, Shapiro SS, Waknitz MA, Swiergiel JJ, Marshall VS, Jones JM. Embryonic stem cell lines derived from human blastocysts. Science 1998;282: 1145-7

White J, Dalton S. Cell cycle control of embryonic stem cells. Stem Cell Reviews 2005;1:131-8

Wianny F, Real FX, Mummery CL, Van Rooijen M, Lahti J, Samarut J, Savatier P. G1-phase regulators, cyclin D1, cyclin D2, and cyclin D3: up-regulation at gastrulation and dynamic expression during neurulation. Dev Dyn 1998;212:49-62 